\title{
Rituximab in Refractory Anti Synthetase Syndrome
}

\author{
Isabel Serrano García ${ }^{1}$, Alba Pérez Linaza ${ }^{1}$, Carolina España ${ }^{2 *}$, Fermín Medina Varo $^{1}$ and Aurelio \\ Arnedillo $^{2}$ \\ ${ }^{1}$ U.G.C. Rheumatology Service of Orthopedic Surgery, Traumatology and Rheumatology, Puerta del Mar University Hospital, Spain
}

${ }^{2}$ Department of Pneumology, Allergy and Thoracic Surgery U.G.C, Puerta del Mar University Hospital, Spain

Corresponding author: Carolina España Domínguez, Department of Pneumology, Allergy and Thoracic Surgery U.G.C., Puerta del Mar University Hospital, Goya Street nํ11, 8D, 11010, Cádiz, Spain

\section{ARTICLE INFO}

Received: 慧 October 12, 2020

Published: 幽 October 23, 2020

Citation: Isabel Serrano García, Alba Pérez Linaza, Carolina España, Fermín Medina Varo, Aurelio Arnedillo. Rituximab in Refractory Anti Synthetase Syndrome. Biomed J Sci \& Tech Res 31(3)-2020. BJSTR. MS.ID.005093.

\section{ABSTRACT}

A 55-year-old woman with a diagnosis of positive Jo1 antisynthetase syndrome with interstitial lung disease began treatment with high doses of corticosteroids and cyclophosphamide, with clinical and radiological progression despite treatment. The therapeutic scheme was modified to rituximab, observing a quick and excellent response to clinical and radiological parameters.

Keywords: Antisynthetase syndrome; Interstitial lung disease; Rituximab

Abbreviations: IIM: Idiopathic inflammatory myopathies; ASS: Antisynthetase Syndrome; ILD: Interstitial Lung Disease; MP: metacarpophalangeal; PIP: Proximal Interphalangeal; CK: Creatinine Kinase; ANA: Antinuclear Antibody; HRCT: High Resolution CT; NIH: National Institute of Healt usual UIP: usual interstitial pneumonia; ASAB: Anti Synthetase Antibodies; EMG: Electromyography; MRI: Magnetic Resonance Imaging; PM: Polymyositis; DM: Dermatomyositis

\section{Introduction}

Idiopathic inflammatory myopathies (IIM) are a group of heterogeneous processes in which there is muscle weakness and inflammation. Antisynthetase syndrome (ASS) is characterized by a variable combination of inflammatory myositis, arthritis, interstitial lung disease (ILD), "mechanic's hands," Raynaud's phenomenon, and fever, along with the presence of some antitRNA synthetase antibody. Treatment of ASS depends on the severity and progression to ILD, often including the combination of corticosteroids and immunosuppressant drugs [1,2]

\section{Case Report}

55-year-old woman ex-smoker of 1 packet per day for over 10 years. She has C6-C7 protrusion, lumbar canal stenosis, granuloma annulare and allergic rhinitis. She suddenly presented arthritis in 1st and 2nd metacarpophalangeal (MP) and proximal interphalangeal (PIP) joints of both hands and carpus, with pain and some functional limitation with occasional dysesthesias. She also presents hyperkeratosis and fissuring of the skin on the radial side of the 2 nd fingers, shoulder girdle weakness, dyspnea on exertion for a month and non-productive cough. She did not report fever, Raynaud's phenomenon, photosensitivity, mucosal ulcers, alopecia or visual disturbances. Pulmonary auscultation shows crackles at the lung bases. Analytically, there was an elevation of muscle enzymes: creatinine kinase (CK): $845 \mathrm{U} / \mathrm{L}$, Aldolase: 56,3 U/L; with normal acute phase reactants. Autoimmunity study: antinuclear antibody (ANA) 1/320 nucleolar pattern, positive antiJo1. Chest CT scan showed an interstitial pneumonia with a suggestive pattern of organized pneumonia associated with ASS (Figure 1A). The diagnosis of positive Jo1 ASS with interstitial lung disease was established, initiating treatment with high doses of corticosteroids and cyclophosphamide in the National Institute of Health (NIH) scheme. Prior to the last administration of cyclophosphamide, the patient worsened clinical and analytically, showing the new high resolution CT (HRCT) scan findings of interstitial pneumonia with a pattern of usual interstitial pneumonia (UIP) that has worsened compared to the previous study, with possible progression to fibrosis (Figure 1B). Therefore, it was decided to start treatment with rituximab, observing an early and excellent response to clinical and radiological parameters (Figure 1C). 


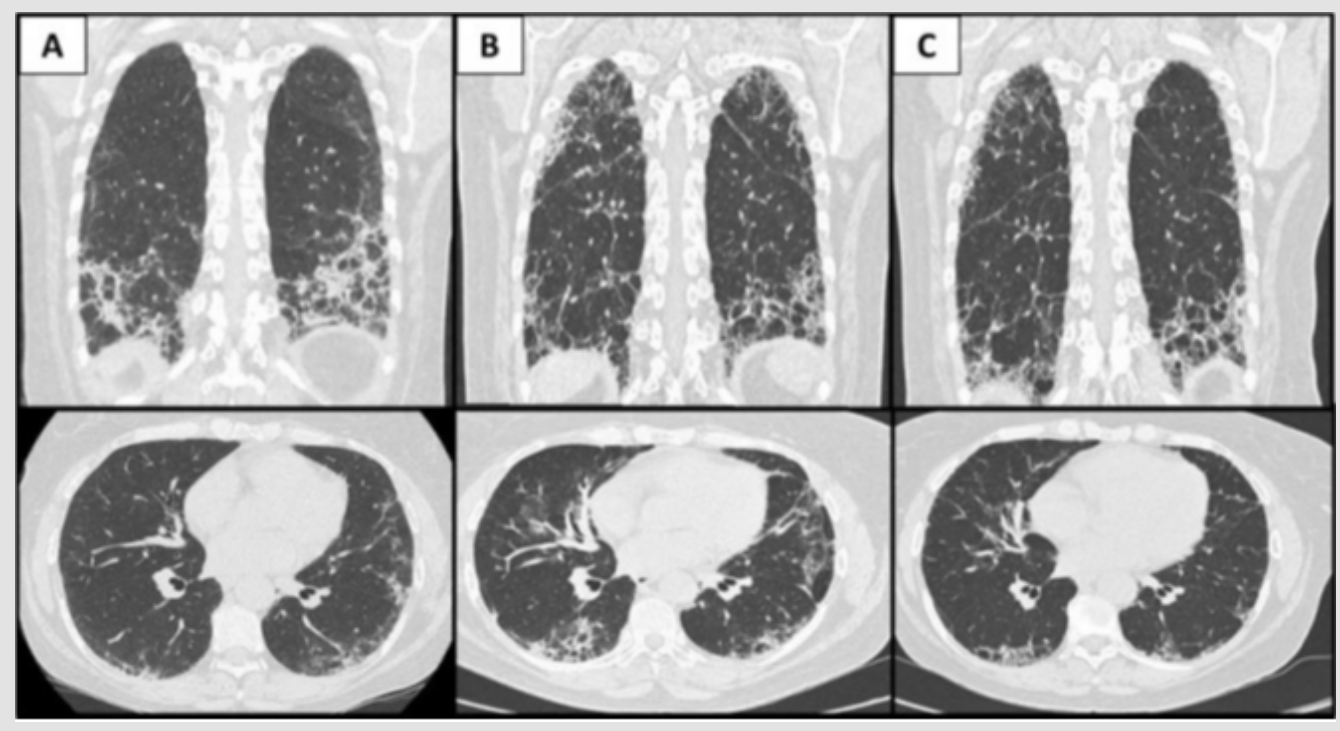

Figure 1: A. Sagittal and axial views of the chest CT scan performed at diagnosis, showing bilateral interstitial involvement consisting of peripheral heterogeneous consolidations that predominate in the lung bases associated with reticulation and ground glass opacities. B. Chest CT scan at the time of radiological worsening despite first-line treatment, a decrease in consolidation images is observed with a greater presence of reticulation, with traction bronchiectasis and greater involvement of the middle and upper fields of both lungs, indicating progression fibrosis. C. Chest CT scan after treatment with rituximab, peripheral crosslinking and traction bronchiectasis continued, the compromise affected to a greater extent the posterior regions of the middle and lower fields of both lungs, having decreased that of the upper fields.

\section{Discussion}

IIMs may affect adults and children and are more common in female subjects (female to male ratio about, $F: M=2: 1$ ). The IIMs are rare diseases, with an annual incidence of 2-7 cases per million inhabitants. ASS is a clinically distinct IIM subset, affecting adults at any age, mainly females ( $\mathrm{F}: \mathrm{M}=3: 1$ ), positive for anti-aminoacyl-transfer RNA synthetase (ARS) antibodies (also called anti synthetase antibodies (ASAb)) and characterized by a highly variable clinical picture [3]. Diagnostic criteria for ASS require one or more $\mathrm{ASAb}$ and one or more of the following three clinical features: ILD, inflammatory myopathy and inflammatory polyarthritis affecting small joints. The clinical presentation is a clue to the diagnosis of ASS. Special investigations help to support the diagnosis. These may include the following depending on the clinical context: muscle enzymes (e.g., CK and aldolase, these are often elevated), ASAb, electromyography (EMG), magnetic resonance imaging (MRI) of affected muscles, muscle biopsy, lung function tests, HRCT scan or lung biopsy [4]. Patients who present features mimicking but atypical for rheumatoid arthritis (RA) (it means, negative cyclic citrullinated peptide antibody and nonerosive arthritis), ASS should be considered due to its potential overlap.

A diagnosis of probable ASS would be met when either ILD and/ or inflammatory is present in a patient with ASAb. Approximately $5-8 \%$ of ASS cases appear as overlap syndromes with another connective tissue disease such as systemic lupus erythematosus
(SLE), systemic sclerosis (SSc) and Sjögren's syndrome [4]. The most frequent anti-ARS antibody is the anti Jo-1, directed against the histidyl-tRNA synthetase, whereas other specificities are less frequently identified (eg, anti-PL7, PL12, OJ, EJ, KS, Ha, Zo) [3]. Differences in clinical spectrum presentation and evolution between anti-Jo1 and non-anti-Jo1 positive ASS should be assessed on large casuistries, even if an isolated ILD seems to be more common in non-anti-Jo1 positive patients. Non anti-Jo1 positivity impacts patients' survival [5]. It is important to remember that anti-Ro antibodies (mainly anti-Ro52 kDa) and anti-ARS antibodies co-occur in about $50 \%$ of cases, and that a cytoplasmic positivity of ANA is commonly observed in ASS [6]. Lung damage is the main cause of mortality in inflammatory myopathies. ASS is an autoimmune inflammatory disease characterized by a worse prediction compared to polymyositis (PM) and dermatomyositis (DM), especially due to the severity of the lung damage that is defined by ILD. A proportion of patients also develop pulmonary arterial hypertension and, less commonly, obstructive bronchiolitis or acute respiratory failure (probably related to the underlying autoantibody pattern) [1,2].

Lung damage changes from mild forms to a rapidly progressive disease that can lead to chronic lung damage if misdiagnosed and it is not properly treated. Therefore, a multidisciplinary approach will be essential as well as carrying out the identification and adequate treatment of associated risk factors [1,2]. Treatment with glucocorticoids and immunosuppressant drugs is used in a 
variable way to treat mild and severe cases. Previous studies are demonstrating the efficacy of rituximab in refractory forms of first-line immunosuppressive treatment and rapidly progressive, improving respiratory function tests and the extent of ILD, as supported by our case $[1,2]$.

\section{References}

1. Gasparotto M, Gatto M, Saccon F, Ghirardello A, Iaccarino L, et al. (2019) Pulmonary involvement in antisynthetase syndrome. Current Opinion in Rheumatology 31(6): 603-610.

2. Barba, T, Mainbourg S, Nasser, M, Lega, J, Cottin V (2019) Lung Diseases in Inflammatory Myopathies. Seminars in Respiratory and Critical Care Medicine 40(02): 255-270.

\section{ISSN: 2574-1241}

DOI: 10.26717/BJSTR.2020.31.005093

Carolina España. Biomed J Sci \& Tech Res

(C) (i) This work is licensed under Creative

Submission Link: https://biomedres.us/submit-manuscript.php
3. Cavagna L, Nuño L, Scirè CA, Govoni M, López Longo FJ, et al. (2015) Clinical Spectrum Time Course in Anti Jo-1 Positive Antisynthetase Syndrome: Results from an International Retrospective Multicenter Study. Medicine (Baltimore) 94: e1144.

4. Cojocaru M, Cojocaru IM, Chicos B (2016) New Insights into Antisynthetase Syndrome. Maedica (Bucur) 11(2): 130-135.

5. Hervier B, Devilliers H, Stanciu R, Meyer A, Uzunhan Y, et al. (2012) Hierarchical cluster and survival analyses of antisynthetase syndrome: phenotype and outcome are correlated with anti-tRNA synthetase antibody specificity. Autoimmun Rev1(2): 210-217.

6. Aggarwal R, Dhillon N, Fertig N, Koontz D, Qi Z, et al. (2017) A Negative antinuclear antibody does not indicate autoantibody negativity in myositis: role of anticytoplasmic antibody as a screening test for antisynthetase syndrome. J Rheumatol 44: 223-229.

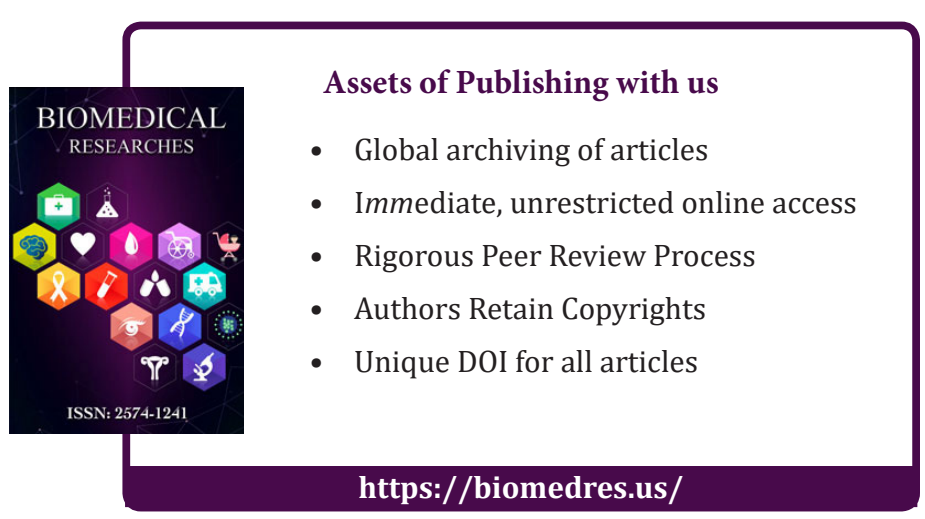

